\title{
An Overview of Long-range Dependent Network Traffic Engineering and Analysis: Characteristics, Simulation, Modelling and Control
}

\author{
Karim Mohammed Rezaul and Vic Grout \\ Centre for Applied Internet Research (CAIR), University of Wales, NEWI, Wrexham, UK \\ \{karim|vic\}@cair-uk.org
}

\begin{abstract}
This paper surveys techniques for the recognition and treatment of self-similar network or internetwork traffic. Various researchers have reported traffic measurements that demonstrate considerable burstiness on a range of time scales with properties of self-similarity. Rapid technological development has widened the scope of network and Internet applications and, in turn, increased traffic volume. The exponential growth of the number of servers, as well as the number of users, causes Internet performance to be problematic as a result of the significant impact that long-range dependent traffic has on buffer requirements. Consequently, accurate and reliable measurement, analysis and control of Internet traffic are vital. The most significant techniques for performance evaluation include theoretical analysis, simulation, and empirical study based on measurement. In this research, we discuss existing and recent developments in performance evaluation and control tools used in network traffic engineering.
\end{abstract}

\section{Keywords}

Self-similarity, ACF, LRD, Heavy-tailed distribution, Hurst parameter.

\section{INTRODUCTION}

Several researchers report that today's network traffic exhibits properties of self-similarity and long-range dependence. Self-similar processes were first identified by Kolmogorov [1] in 1941. These processes were then brought to the attention of statisticians by Mandelbrot and colleagues in the late 1960's and early 1970's [2, 3, 4, 5]. Mandelbrot outlined the important dynamic characteristic of fractional Gaussian noises ( $f G n$ ), characteristics which could be quantified with the Hurst exponent. The ordering of such series determined the dynamic measures (i.e. long-range dependence or LRD). Self-similar and LRD characteristics of Internet traffic have attracted the attention of researchers since 1994, when it was discovered that some aspects of LAN traffic exhibits selfsimilar, rather than Poisson, behaviour [6].

Permission to make digital or hard copies of all or part of this work for personal or classroom use is granted without fee provided that copies are not made or distributed for profit or commercial advantage and that copies bear this notice and the full citation on the first page. To copy otherwise, or republish, to post on servers or to redistribute to lists, requires prior specific permission and/or a fee.

Valuetools'07, October 23-25, 2007, Nantes, France.

Copyright 2007 ICST 978-963-9799-00-4
LRD is of great significance in traffic engineering problems such as measurement, queuing strategy buffer sizing and admission and congestion control. In [7] it is shown that consequences of LRD are packet and application level delays that cause a heavy-tailed distribution. The Transmission Control Protocol (TCP) estimates round trip timer values from peer acknowledgements and, as a result, congestion appears more frequently when maintaining impulsive behaviour with an increase in load. The influence of LRD properties on delay at packet and application level is reported in [8]; metrics of network performance, such as throughput, packet loss, latency and buffer occupancy levels, are affected by the presence of LRD phenomenon across many types of networks. The impact of LRD on Quality of Service $(Q o S)$ is analysed in [9] showing that, the greater the LRD, the lower the QoS.

The LRD property of traffic fluctuations has important implications for the performance, design and dimensioning of the network [6]. A simple, direct parameter, characterizing the degree of LRD, is the Hurst parameter. The Hurst exponent (or Hurst parameter, H), which more than a half-century ago was proposed for the analysis of reservoir long-term storage capacity [10], is used today to measure the intensity of LRD in network traffic. A number of methods have been proposed to estimate the Hurst parameter. Some of the most popular include aggregated variance time (V/T) [11], Rescaledrange $(\mathrm{R} / \mathrm{S})[6,10]$ and the Higuchi and wavelet-based methods $[12,13]$ although there are many others. In all these methods, $\mathrm{H}$ is calculated by taking the slope from a log-log plot. Over time, the wavelet-based Hurst parameter has acquired popularity in estimating LRD traffic. The intensity of long-range dependence can be measured for file or document size [14], packet-count [15], inter-arrival time [16], frame size [17], connection size [18], packet length [19], byte-count [6], and bit or byte rate [20] amongst others.

A number of factors, such as a slow start phase of the congestion window, packet losses, ack-compression of TCP traffic and multiplexing of packets at the bottleneck rate, can cause either short- or long-term burstiness in the 
behaviour of TCP flow [21]. The research in [22] investigates how various versions of TCP congestion control affect network performance when traffic is bursty. TCP represents the dominant transport protocol of the network (e.g. Internet), which contributes to the propagation of self-similarity. It is shown in [23] that TCP itself inherits self-similarity when it is combined with self-similar background traffic in a bottleneck buffer through the transform function of the linear system.

This paper is organised as follows. Section 2 describes the relationship between self-similarity, long-range dependence and the autocorrelation function $(A C F)$. Section 3 discusses the performance of the estimators and their reliability. Section 4 explains heavy-tailedness in traffic patterns. Section 5 considers the issues involved in controlling network traffic. Section 6 gives a brief account of modelling the network traffic.

\section{THE RELATIONSHIP BETWEEN SELF-SIMILARITY, LRD AND ACF}

A phenomenon that is self-similar looks or behaves the same when viewed at different degrees of magnification or different scales on a given dimension and is bursty over all time scales. Figure 1 depicts self-similarity observed in physical or natural phenomena such as fern leaves in a branch, sunflowers in a plot, scheme of carpet, human being itself when gathering etc. The bottom left shows a higher magnification of a small segment of an image that demonstrates self-similarity. This type of self-similarity at all scales characterises the fractal nature of an image. Thus, self-similar data have a common property with fractals: when we zoom in on part of the traffic generated by the data, we observe the same structure. Many successive zooms show the same result [24] as shown in the bottom right.

Self-similarity is the property of a series of data points to retain a pattern or appearance regardless of the level of granularity used and is the result of LRD in the data series. If a self-similar process is bursty on a wide range of timescales, it may exhibit LRD. Often lagged autocorrelations are used in time series analysis for empirical stationary tests. Self-similarity manifests itself in the time series of arrivals' LRD (i.e., long memory). The evidence of very slow, linear decay in the sample lag ACF indicates nonstationary behaviour [25]. Long-rangedependence means that all the values at any time are correlated in a positive and non-negligible way with values at all future instants.

A continuous time process $Y=\{Y(t), t \geq 0\}$ is self-similar if it satisfies the following condition:

$$
Y(t) \stackrel{d}{=} a^{-H} Y(a t), \quad \forall a>0, \text { and } 0<H<1
$$

[26] where $\mathrm{H}$ is the index of self-similarity (the Hurst parameter) and the equality is in the sense of finitedimensional distributions.

The stationary process $\mathrm{X}$ is said to be an LRD process if its ACF is non-summable [27], that is if $\sum_{k=-\infty}^{\infty} \rho_{k}=\infty$

Details of how the ACF decays with $\mathrm{k}$ are of interest because the behaviour of the tail of the ACF completely determines its summability. From [6], $\mathrm{X}$ is said to exhibit long-range dependence if

$\rho_{k} \sim L(t) k^{-(2-2 H)}$, as $k \rightarrow \infty$

where $\frac{1}{2}<H<1$ and $L($.) slowly varies at infinity, i.e., $\lim _{t \rightarrow \infty} \frac{L(x t)}{L(t)}=1$, for all $x>0$.

Equation (2) implies that the LRD is characterized by an ACF that decays hyperbolically rather than exponentially fast.

LRD processes then are characterised by a slowly decaying covariance function that is non-summable. Numerous studies [6, 28, 29] of different types of data networks (LANs \& WANs) and services/applications (ATM, Frame Relay, WWW, etc.) have shown that aggregated traffic at packet level exhibits slowly decaying autocorrelation that leads to LRD. The periodic nature of the traffic leads to high and slowly decaying autocorrelation [30]. When network performance is affected by LRD, data are correlated over an unlimited range of time lags and this property results in a scale invariance phenomenon. Then no characteristic time scale can be identified in the process: they are all equivalent for describing its statistics - the part resembles the whole and vice versa. LRD and self-similarity are not equivalent. A random process is self-similar if $0<H<1$. However, the process is both LRD and self-similar when $0.5<H<1$. Therefore, all LRD processes are self-similar, but all selfsimilar processes do not hold the properties of LRD.

In Figure 2, the top left plot illustrates typical LRD and SRD (short-range dependent) processes. The top right plot shows traffic burstiness for real data. Having positive correlations of data (bit/second), the ACF plot at the bottom confirms that the traffic is LRD. Clearly, traffic persists for more than 9 seconds. These real data were taken from the UNC archive [31] with trace collections obtained from a Gigabit Ethernet link. The data set considered here are number of TCP bytes per $10 \mathrm{~ms}$ bin. The sample length $(\mathrm{N})$ considered for the dataset is 10,000 . 


\section{FINDING RELIABLE AND ROBUST ESTIMATORS}

Several studies report problems with existing estimators, such as the unreliability of the wavelet-based Hurst parameter [32, 33, 34, 35], R/S analysis [36, 37], aggregated V/T analysis [38, 39, 40], Whittle estimator
[33, 41] and Periodogram analysis [39]. The estimated Hurst parameters from the wavelet analysis, aggregated variance and Whittle method have been presented [33] for both real and synthetic data.
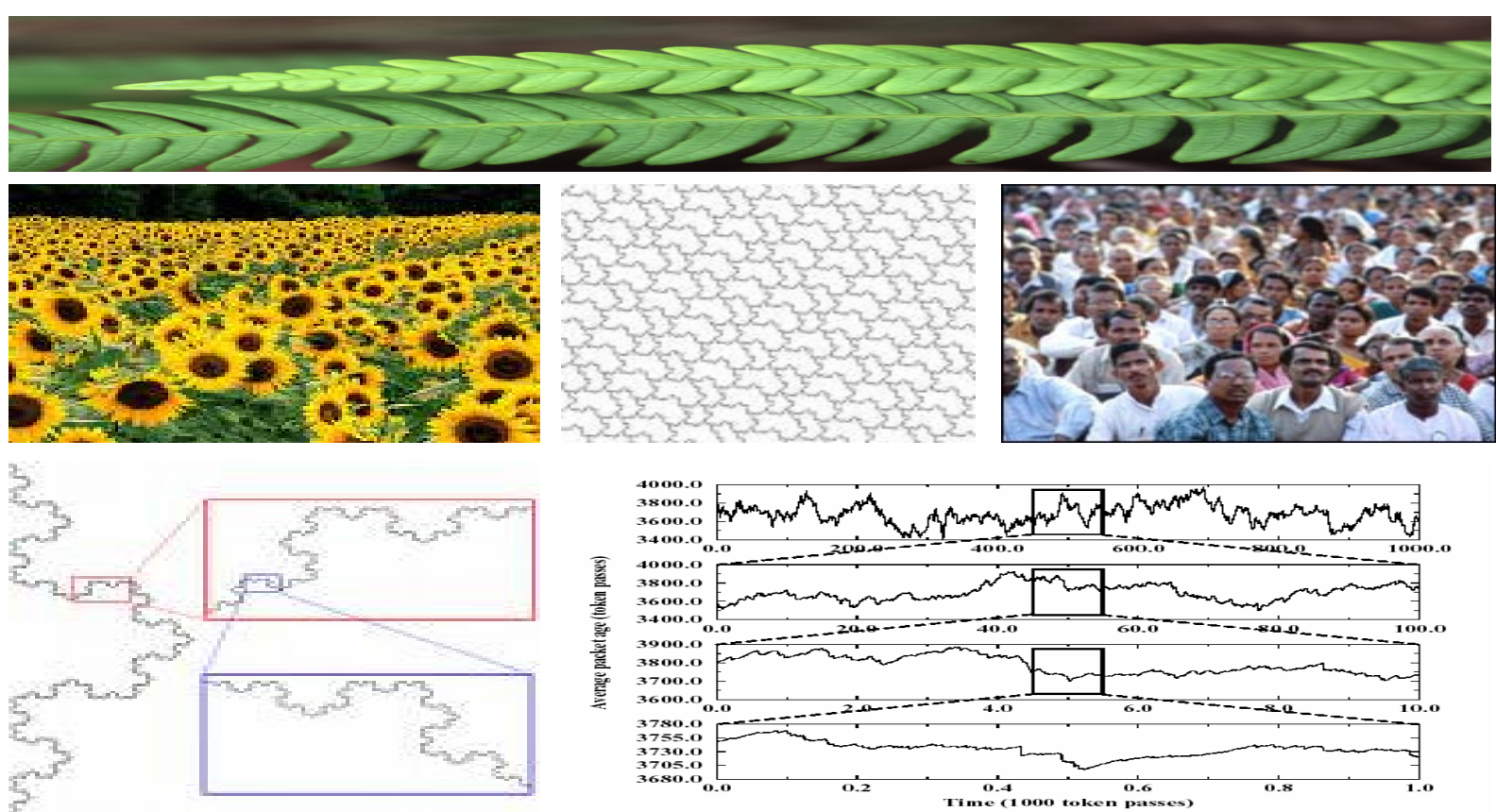

Figure 1. Self-similar phenomena
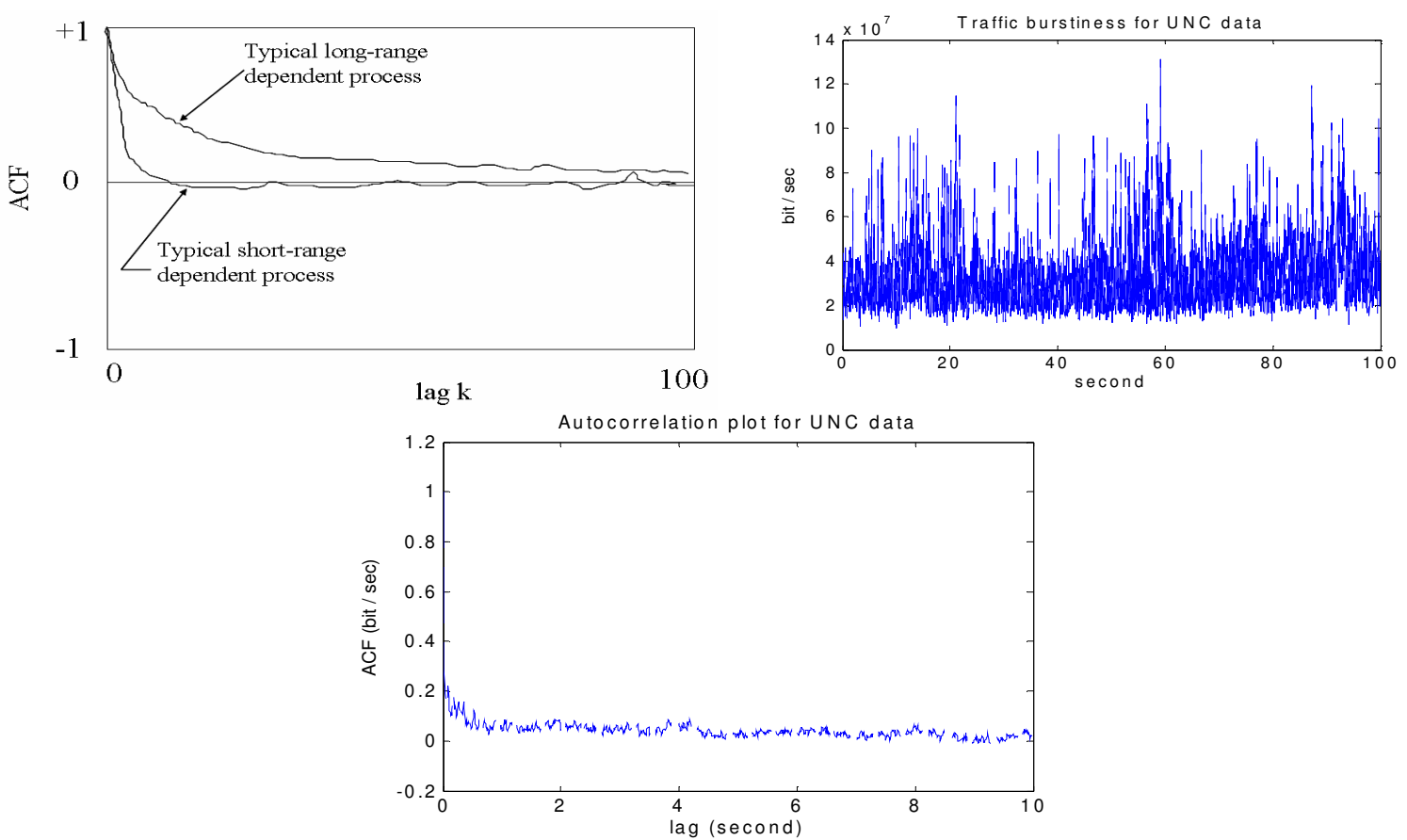

Figure 2. Autocorrelation plot and burstiness of network traffic 
It is observed that the Whittle and wavelet methods overestimate the degree of self-similarity (i.e., produce $H>1$ ). In [34] the advantages and limitations of the wavelet estimators are explored. Here it is found that a
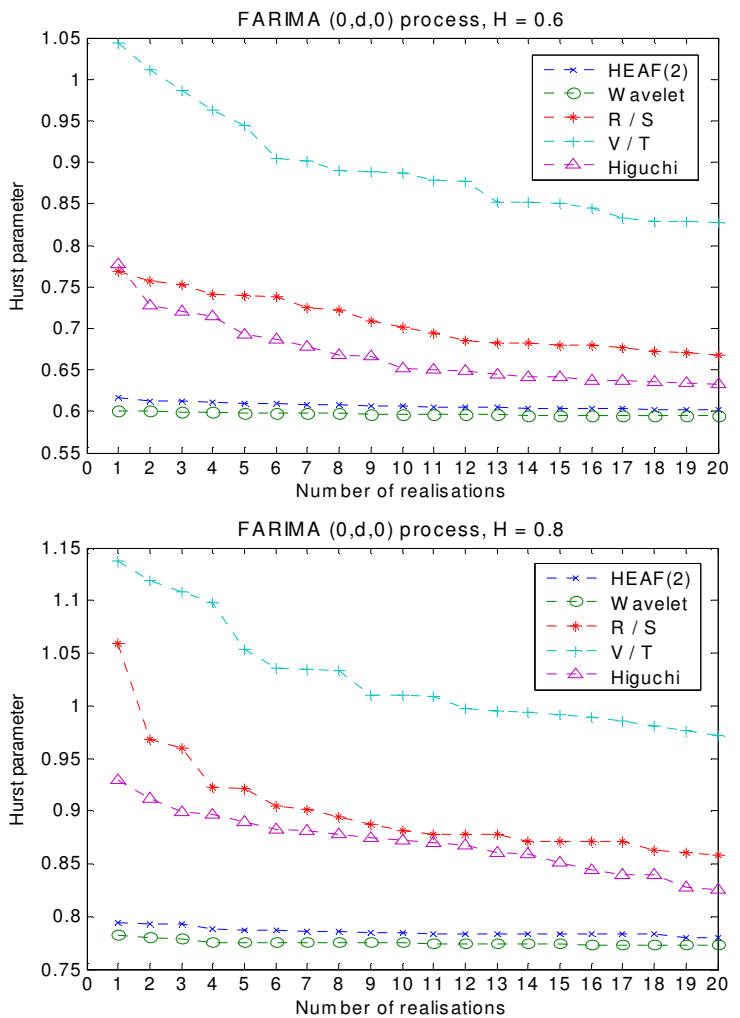

traffic trace with a number of deterministic shifts in the mean rate results in a steep wavelet spectrum, which leads to overestimating the Hurst parameter.
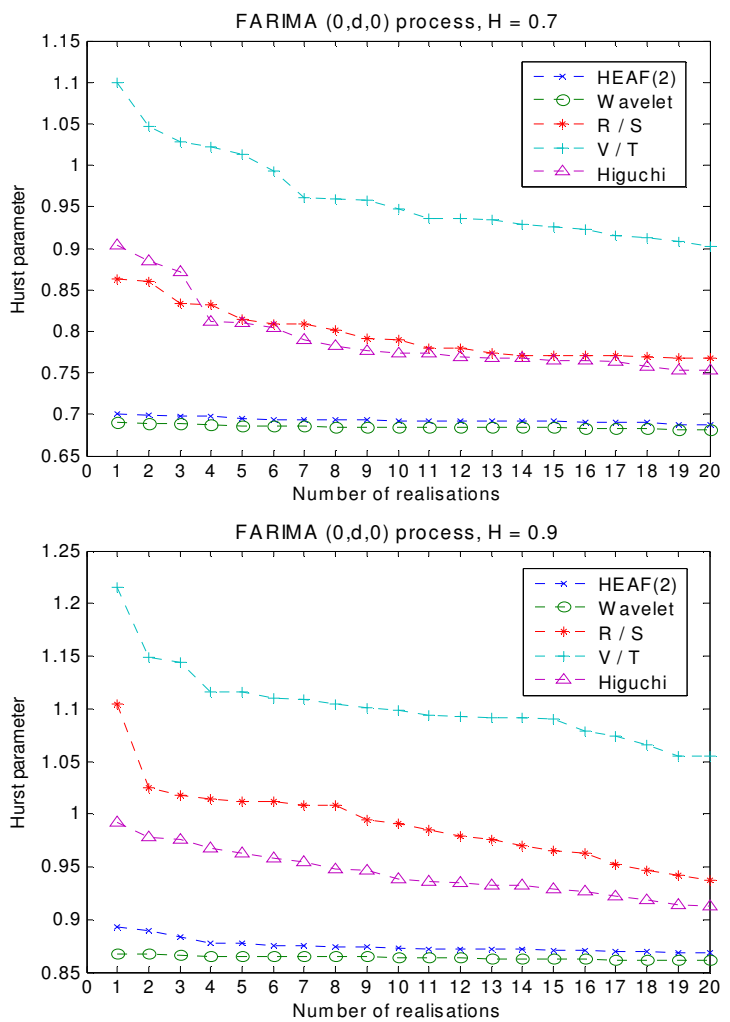

Figure 3. Reliability of the estimators. A simulation experiment using the FARIMA(0, d, o) process. Highest Hvalues for 20 different realisations (out of 100 realisations). Sample length $N=16384$. [43]

In [42] it is reported that the interpretation of the estimated Hurst parameter is problematic in practice. Numerous studies have shown that existing estimators provide an unreliable estimate of the Hurst parameter $(\mathrm{H})$. Having a reliable estimator can yield a good insight into traffic behaviour and should eventually lead to improved traffic engineering. Figure 3 compares the reliability of some popular estimators. Here the 20 highest values of the Hurst parameter provided by different estimators are shown while the self-similar sequences were generated by a fractional autoregressive integrated moving average (FARIMA) process for a particular Hurst parameter, $\mathrm{H}$ [43].

It is possible to derive wrong conclusions and wrong models when measuring the intensity of the LRD with unreliable estimators. In $[44,45]$ an estimator is introduced called the Hurst Exponent from the Autocorrelation Function (HEAF) and it is shown that (and why) lag 2 in HEAF (i.e. HEAF (2)) is considered when estimating LRD of network traffic. [43] considers the robustness of $\operatorname{HEAF}(2)$ when estimating the Hurst parameter of data traffic (e.g. packet sequences) with outliers and also the reliability of $\operatorname{HEAF}(2)$. Also, based on the comparison of simulation experiments shown [43, $44,45]$ for both fGn and FARIMA $(0, \mathrm{~d}, 0)$ processes, it is evident that $\operatorname{HEAF}(2)$ is a stable method that quantifies the reliable degree of LRD. Through its simplicity, robustness and reliability, $\operatorname{HEAF}(2)$ can be used to estimate the intensity of LRD in real time network traffic. 


\section{HEAVY-TAILEDNESS IN TRAFFIC PATTERNS}

Self-similarity and heavy-tailedness are of great importance for network capacity planning purposes, in which researchers are interested in developing analytical methods for analysing traffic characteristics. The goal of traffic characterisation is to determine the nature of the traffic and develop tractable models that capture the important properties of data, which can eventually lead to accurate performance prediction. The uses of traffic characterisation include network planning, design, capacity management, performance prediction, real-time traffic management and network control. The terms longrange dependent, self-similar and heavy-tailed are relatively close in meaning. In fact, superpositions of samples from heavy-tailed distributions aggregate to form long-range dependent time series.
Many researchers have discussed the effects of heavytailedness in network traffic patterns and shown that Internet traffic flows exhibit characteristics of selfsimilarity that can be explained by the heavy-tailedness of the various distributions involved. The properties of heavy-tailed distributions are qualitatively different to commonly used memoryless distributions such as the exponential, normal or Poisson distributions. [46] concludes that such exponentiality assumptions mislead exploration of the presence of heavy-tailed distributions. The condition of self-similarity is that the ACF of the time-series declines as a power-law, leading to positive correlations among widely separated observations [47]. In the Internet, heavy-tailed distributions have been observed in the context of traffic characterization and self-similarity is mainly caused by the heavy-tailed nature of the file sizes transferred. The distributions having infinite variances are called heavy-tailed and the weight of their tails is determined by a parameter called the tail index, $\alpha<2$ [48].
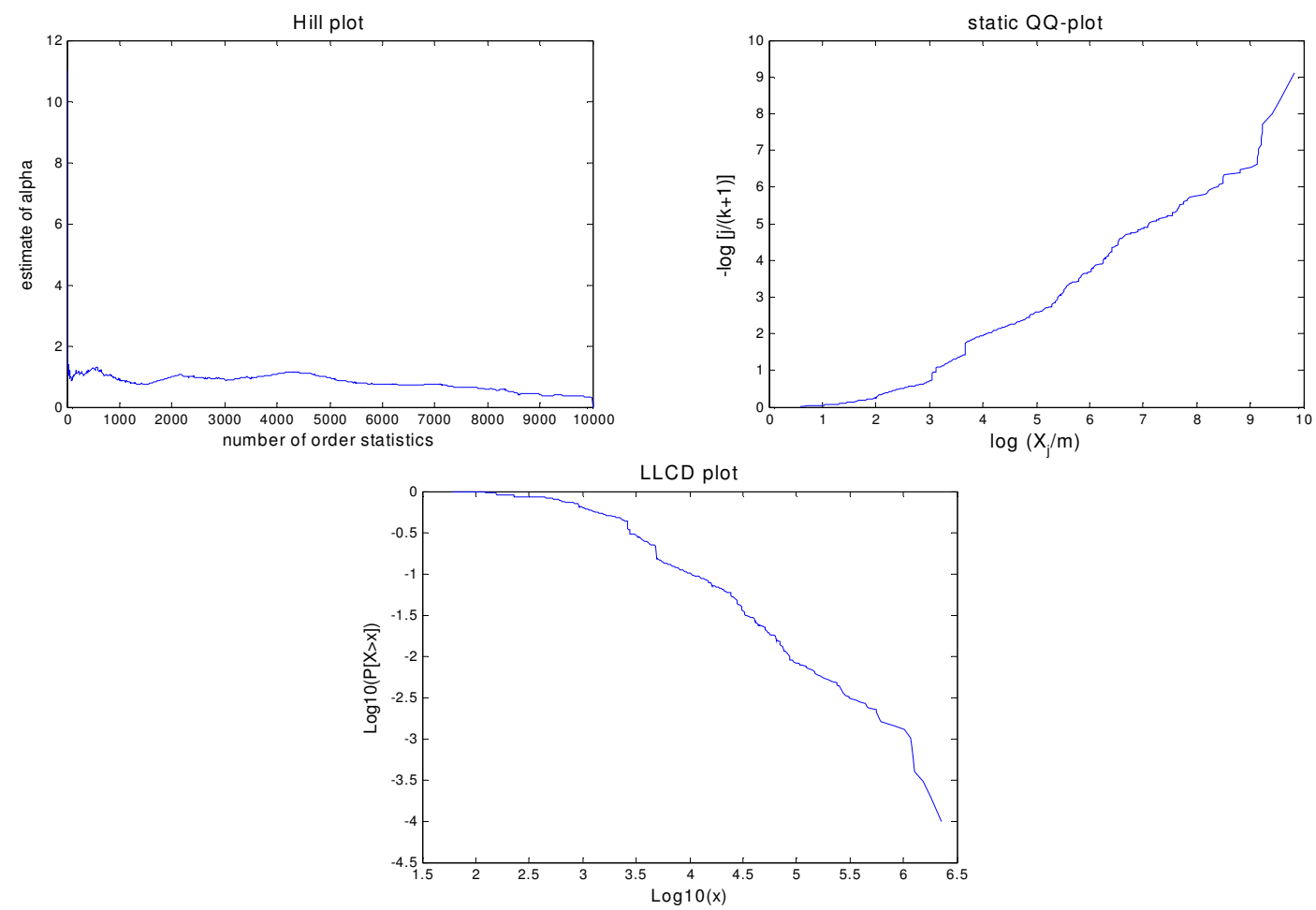

Figure 4. Estimation of tail index by Hill plot ( $\alpha=0.76)$, static-qq plot ( $\alpha=0.74)$ and LLCD plot $(\alpha=0.80)$ [49]

The performance of several estimators of the tail index $(\alpha)$ for heavy-tailed Internet traffic have been studied in [49]. A summary of results is shown in Figure 4. In most cases, the moment estimator due to an unstable region is observed in the graph. The Hill plot, static qq plot and LLCD plot show a good level of agreement when estimating the index from graphs. The results show that there are infinite variances (i.e. $\alpha<2$ ) observed in the traffic, which is indicative of the existence of heavytailedness in the Internet traffic.

[50] proposes some analytical models based on the Empirical Distribution Function (EDF) statistics, which can characterise web traffic. The analyses show that the 
Weibull (three parameters) and generalized Pareto distributions (GPD), with the experimental results, are the most suitable to approximate the traffic, as shown in Figure 5. In addition, the generalized Pareto model (GPD) is more suitable for analysing traffic behaviour than the simple Pareto model in terms of heavy-tailedness. Hence, as an efficient analytical tool, the generalised Pareto model can be used for identifying a heavy-tailed nature based on samples from web traffic.
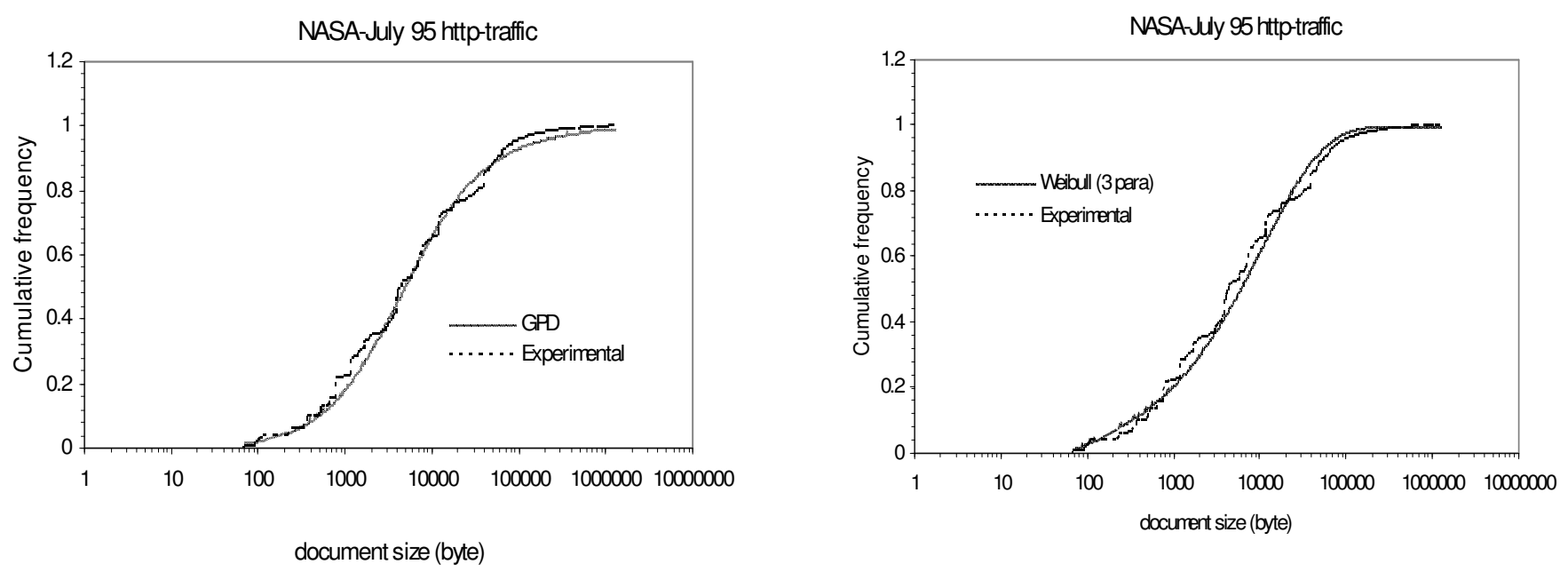

Figure 5. Web file size cumulative distribution of different analytic models for NASA (http-95-August) traffic [50]

\section{CONTROLLING NETWORK TRAFFIC}

Most research in this area concentrates on modelling network traffic rather than controlling it. However, controlling network traffic should not be overlooked as it can help reduce the network load and lead to the improvement of QoS in future network performance. [51] introduces a novel algorithm, called CoLoRaDe, to control the intensity of LRD traffic. Experimental results show that CoLoRaDe is capable of reducing the LRD of packet sequences received at the router buffer before they are transmitted to the core network (i.e. the Internet). As the main function of the CoLoRaDe algorithm is to reduce the LRD of packet traffic, it can contribute in reducing the network load, leading to an improvement in QoS for the Internet of the future.

A number of factors, such as a slow start phase of the congestion window, packet losses, ack-compression of TCP traffic and multiplexing of packets at the bottleneck rate, can cause either short- or long-term burstiness in TCP flow [52]. [53] investigates how various versions of TCP congestion control affect network performance when traffic is bursty. A significant adverse impact on network performance is shown, attributable to traffic selfsimilarity and, while throughput declines gradually as self-similarity increases, queueing delay increases more drastically. TCP represents the dominant transport protocol of the Internet, which contributes to the propagation of self-similarity [23].

Various researchers have reported that traffic measurements demonstrate considerable burstiness on several time scales, with properties of self-similarity. Bursty traffic can affect the QoS for all traffic on the network by introducing inconsistent latency. It is easier to manage the workloads under less bursty (smoother) conditions. One of the major drawbacks of TCP/IP is the lack of true QoS functionality. QoS in networks, in simple terms, is the ability to guarantee and limit bandwidth appropriately for certain services and users. Traffic shaping is an attempt to control network traffic in order to optimize, attempt to optimize or guarantee performance, low-latency or bandwidth, and deals with concepts of classification, queue disciplines, enforcing policies, congestion management, QoS and fairness. [54] introduces a novel algorithm, BPTraSha, to control the bursty nature of network traffic. Experimental results show that the BPTraSha algorithm is capable of smoothing out the bursty nature of traffic packets received at the router buffer before they are transmitted to the core network. Figure 6 shows how the bursty nature of traffic (left figure) are smoothed out (right figure) by BPTraSha algorithm. 

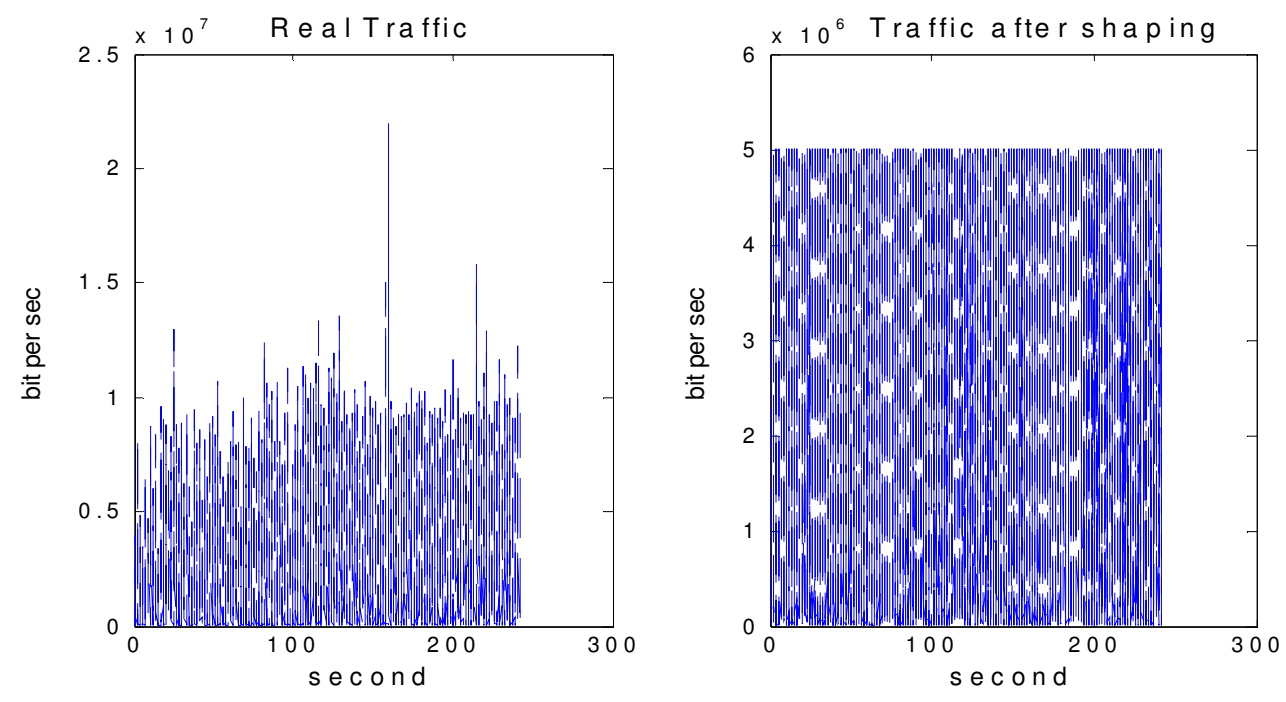

Figure 6. Traffic shaping: LBL-tcp3-pkt, $C=5$ Mbps, Number of samples $(N)=65536$ [54]

\section{MODELLING NETWORK TRAFFIC}

Traffic models play a significant role in the analysis and characterisation of network traffic and network performance. Accurate models can provide the information of complicated signals and system performance through both analysis and simulation. Researchers have made a great progress in modelling network traffic by introducing an idea of fractal. In this case, Fractional Brownian Motion (FBM) has been broadly used to model fractal random processes $[55,16$, 56]. FBM is a generalised version of Brownian motion or Wiener process and an increment process of FBM is called Fractional Gaussian Noise (FGN). These FBM and FGN models are based on Gaussian distribution, which means that there must exist some negative signals. However, the data of network traffic should not be less than zeros. A Fractional Autoregressive Integrated Moving Average (FARIMA) process is used to model self-similar network traffic. FARIMA is a generalisation of ARIMA process, can capture both short-range dependence (SRD) and long-range dependence at the same time.

Fractional Gaussian noise $X=\left(X_{k}: k=0,1,2, \ldots\right)$ with parameter $H \in(0,1)$ is a stationary Gaussian process with mean $\mu$, variance $\sigma^{2}$, and autocorrdation function $r(k)=l / 2\left(|k+1|^{2 H}-2|k|^{2 H}+|k-1|^{2 H}\right), k>0$. Simple calculations show that fractional Gaussian noise is exactly second-order self-similar with self-similarity parameter $H$, as long as $1 / 2<H<1$. Methods for estimating the three unknown parameters $\mu, \sigma^{2}$ and $H$ are known. Fractional ARIMA(p, d, q) processes are a natural generalization of the widely used class of Box-Jenkins models [57] by allowing the parameter $d$ to take noninteger values. They were introduced by Granger and Joyeux [58] and Hosking [59] who showed that fractional $\operatorname{ARIMA}(p, d, q)$ processes are asymptotically secondorder self-similar with self-similarity parameter $d+1 / 2$, as long as $0<d<1 / 2$. Fractional ARIMA processes are much more flexible with regard to the simultaneous modeling of the short-term and long-term behavior of a time series than fractional Gaussian noise, mainly because the latter, having only the three parameter $\mu, \sigma^{2}$ and $H$, has a very rigid correlation structure and is not capable of capturing the wide range of low-lag correlation structures encountered in practice. This flexibility can already be observed when considering the simplest processes of the $\operatorname{ARIMA}(p, d, q)$ family, namely the two-parameter models $\operatorname{ARMA}(1, d, 0)$ and $\operatorname{ARMA}(0, d, 1)($ see $[59])$.

As a modelling tool, multifractal stochastic model has been suggested by the researchers $[55,60,61]$ to understand the complex long-range dependence (LRD) and memory structure in data traffic and for measurement analysis, multiscale techniques such as wavelets have been outlined. Also Fractal Point Processes (FPP) are used in [62] for systematic analysis, modelling and synthesis of point processes with fractal characteristics. Researchers [63, 64, 65] used $M / G / \infty$ model to analyse the performance of network traffic. An $M / G / \infty$ input process is the busy server process of a discrete-time infinite server system fed by a discrete-time Poisson process of rate (customers/slot) and with generic service time (expressed in number of time slots) distributed according to G. The process was studied early 
Data Collection from Traffic Traces

file size or document size, packet counts, inter-arrival time, frame size, connection size, packet length, number of bytes per unit time, Bit or byte rate etc.

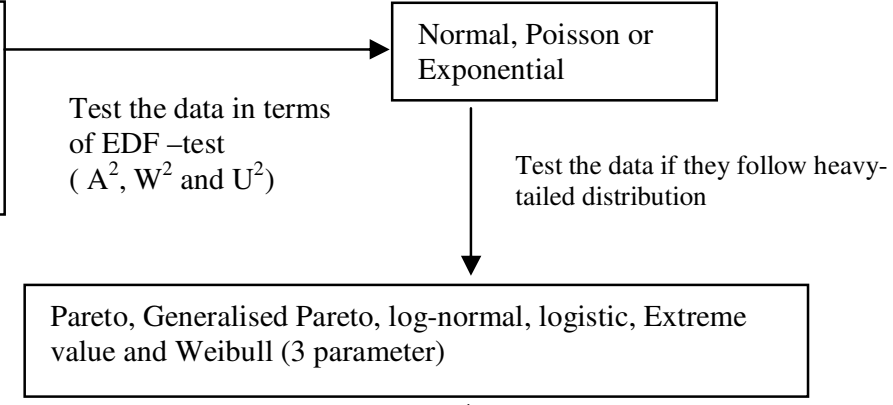

Properties of Self-similar Long-range dependent processes

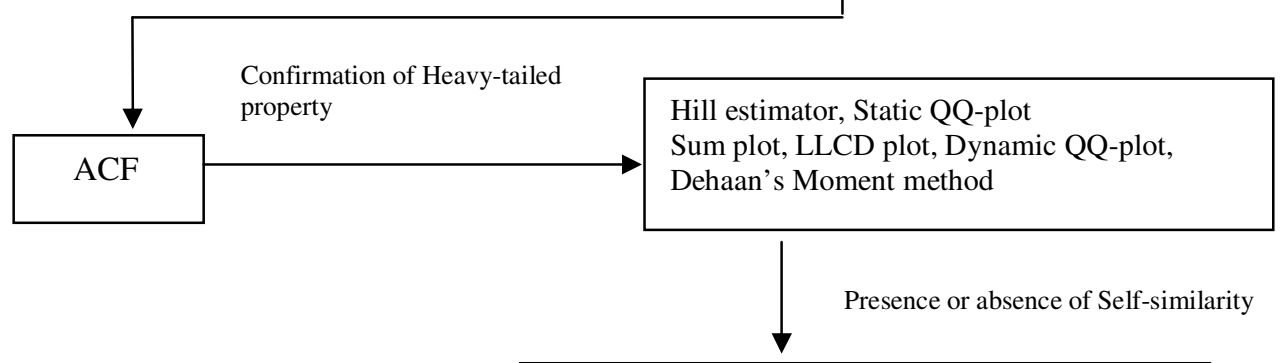

Relationship between Heavy-tailed and LRD

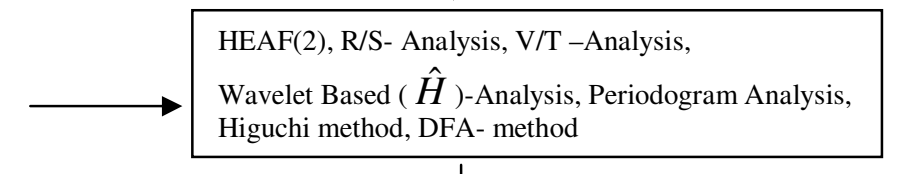

ACF - Autocorrelation Function

FFT - Fast Fourier Transformation

FBM - Fractal Brownian Motion

FGN - Fractal Gaussian Noise

FARIMA - Fractional Autoregressive

Integrated Moving Average

$\mathrm{R} / \mathrm{S}$ - Rescale Range

FGN-DW - FGN and Daubechies Wavelet

FPP - Fractal Point processes

EDF - Empirical Distribution Function

$\hat{H}$ - Estimator of Hurst parameter

$\mathrm{V} / \mathrm{T}$ - Variance-Time

S-S - Self-similar

DFA - Detrended Fluctuation Analysis

MLE - Maximum Likelihood Equation

LRD - Long -Range Dependence

LLCD - Log-log complementary

distribution

QQ - Quantile-Quantile

RMD - Random Midpoint Displacement
Modelling and Fractal random walk simulation (generators of S-S traffic)

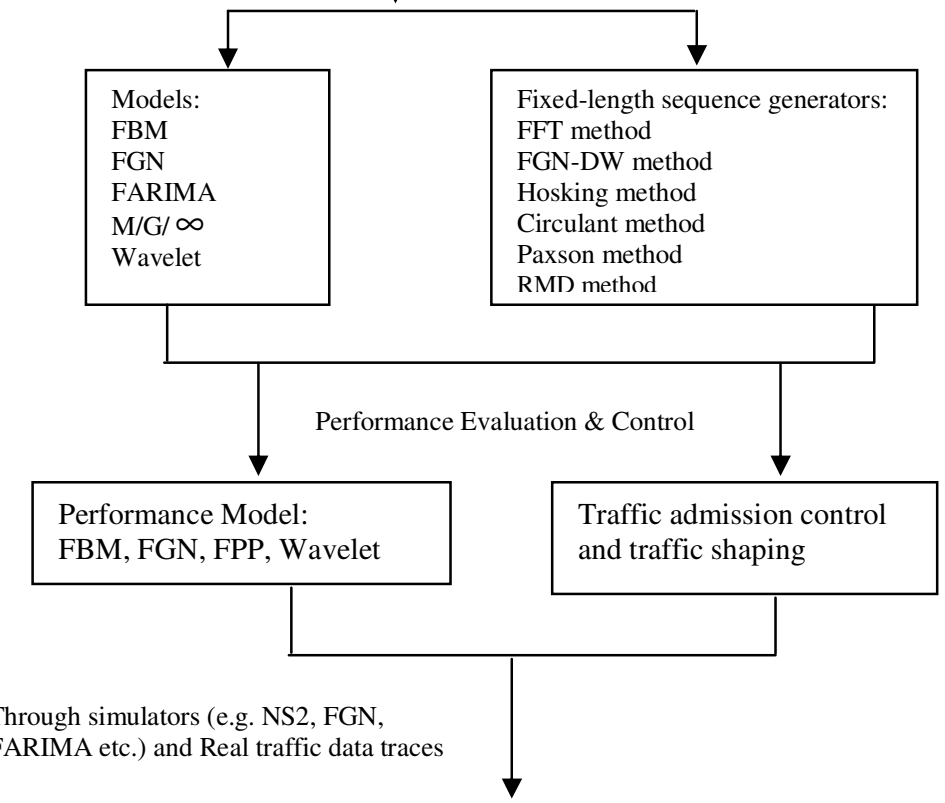

Validation of Model/Method/Algorithm

Figure 7: Traffic engineering process: Characteristics, Simulation, Modelling and Control 
on by Cox [27, 66] as a model for textile yarn processing. His analysis indicated that it was extremely versatile, capable of exhibiting correlations over a wide range of time scales simply by controlling the tail behavior of the distribution of the service time.

An overview of Traffic Engineering and Analysis (Characteristics, Simulation, Modelling and Control) has been shown in Figure 7. Due to space limitation we cannot provide the explanation of this Figure. However it shows a clear view of the network traffic engineering process. We believe that this picture can motivate the new researchers to work in this area of research. Note that the steps in Figure 7 do not necessarily indicate the exact sequential process.

\section{CONCLUSION}

Network performance evaluation is important for assessing the effectiveness of traffic methods and for monitoring and verifying compliance with network performance goals. Results from performance evaluation can be used to identify existing problems, guide network re-optimization and aid in the prediction of potential future problems. Research towards finding and improving suitable tools which may help to characterise various types of network traffic is consequently, and obviously, vital. It is particularly important to understand the link between the self-similarity and long-range dependence of network traffic and the performance of the networks because such characterization can be potentially applied for essential control purposes such as traffic shaping, load balancing and other strategies of the future.

\section{REFERENCES}

[1] Kolmogorov, A. N., The local structure of turbulence in incompressible viscous fluid for very large Reynolds numbers, $C$. R. Acad. Sci. URSS (N. S.), Vol. 30, 1941, pp. 301-305. [Translation in Turbulence (S. K. Friedlander and L. Topper, eds.) 151-155. (1961). Interscience, New York]

[2] Mandelbrot B.B., Self-similar error clusters in communications systems and the concept of conditional stationarity. IEEE Transactions on Communications Technology, 1965, COM-13, pp. 71-90.

[3] Mandelbrot B.B. and Van Ness J. W., Fractional Brownian motions, fractional noises and applications. SIAM Review: 10, 1968, pp. 422-437.

[4] Mandelbrot B.B. and Wallis J. R., M \& James R. WALLIS, Some long-run properties of geophysical records. Water Resources Research: 5, 1969, pp. 321-340.

[5] Mandelbrot B.B. and James R. WALLIS, Noah, Joseph and operational hydrology. Water Resources Research: 4, 1968, pp. 909-918.

[6] Will E. Leland, Murad S. Taqqu, Walter Willinger, Daniel V. Wilson, On the self-similar nature of Ethernet traffic (extended version), IEEE/ACM Trans. Networking, Vol. 2, No. 1, February 1994, pp. 1-15.

[7] Willinger, W., Paxson, V. and Taqqu, M.S., Self-Similarity and Heavy-tails: Structural Modeling of Network Traffic, A Practical
Guide to Heavy Tails, 1998, Birkhauser, Boston.

[8] Jena Ajit K. and Popescu Adrian, Traffic engineering for Internet applications, SPIE proceedings series, Internet Performance and Control of Network Systems II, Robert D. van der Mei; Frank Huebner-Szabo de Bucs; Eds., Vol. 4523, 2001, pp. 67-78.

[9] Philippe Owezarski, Nicolas Larrieu, Measurement Based Approach of Congestion Control for enforcing a robust QoS in the Internet, Report LAAS No. 04722, International Conference on Internet Surveillance and Protection (ICISP'06), Cap Esterel (France), 27-29 August 2006.

[10] Hurst H. E., Long-term storage capacity of reservoirs, Transactions of the American Society of Civil Engineers, 1951, vol.116, pp 770-808.

[11] Ton Dieker, Simulation of Fractional Brownian Motion, Masters Thesis, Department of Mathematical Sciences, University of Twente, The Netherlands, 2004.

[12] T. Higuchi, Approach to an irregular time series on the basis of the fractal theory, Physica D, 1988, vol.31, pp. 277-283.

[13] P. Abry, P. Flandrin, M. S. Taqqu and D. Veitch, Wavelets for the Analysis, Estimation, and Synthesis of Scaling Data, K. Park and W. Willinger (editors), Self-Similar Network Traffic and Performance Evaluation. John Wiley \& Sons, New York, 2000, pp. 39-88.

[14] Kihong Park, Gitae Kim, Mark Crovella, On the relationship between file sizes, transport protocols, and self-similar network traffic, Fourth International Conference on Network Protocols (ICNP'96). 1996, pp. 171-180.

[15] Vern Paxson and Sally Floyd, Wide-Area Traffic: The Failure of Poisson Modeling, IEEE/ACM Transactions on Networking, June 1995, Vol. 3 No. 3, pp. 226-244.

[16] Ashok Erramilli, Onuttom Narayan, and Walter Willinger, Experimental Queueing Analysis with Long-range Dependent Packet Traffic, IEEE/ACM Transactions on Networking, April 1996, Vol. 4, No. 2, pp. 209-223.

[17] O. Rose, Estimation of the Hurst Parameter of Long-Range Dependent Time Series, Report No. 137, February 1996, Institute of Computer Science, University of Wurzburg.

[18] E. Willekens and J. Teugels, Asymptotic expansions for waiting time probabilities in an M/G/1 queue with longtailed service time, Queueing Systems 10, 1992, pp. 295-312.

[19] An Ge, Franco Callegati, and Lakshman S. Tamil, On Optical Burst Switching and Self-Similar Traffic, IEEE Communications Letters, March 2000, Vol. 4, No. 3, pp. 98-100.

[20] R.J. Gibbens, Traffic characterisation and effective bandwidths for broadband network traces, Stochastic Networks, Theory and Applications, 1996, pp. 169-179, Oxford Science Pub.

[21] Amit Aggarwal, Stefan Savage and Thomas Anderson, Understanding the Performance of TCP Pacing. Proc. of the IEEE INFOCOM 2000 Conference on Computer Communications, March 2000, pp. 1157 - 1165.

[22] K. Park, G. Kim, and M. Crovella, On the effect of self-similarity on network performance, In Proceedings of the SPIE International Conference on Performance and Control of Network System, November 1997, pp 296-310.

[23] A. Veres, Zs. Kenesi, S. Molnár, G. Vattay, TCP's Role in the Propagation of Self-Similarity in the Internet, Computer Communications, Special issue on Performance Evaluation of IP Networks and Services, Vol. 26, Issue 8, May 2003, pp. 899-913.

[24] J. H. B. Deane, C. Smythe, and D. J. Jefferies. Self-similarity in a deterministic model of data transfer, International Journal of Electronics, 80(5), 1996, pp. 677-691.

[25] Brocklebank J. and D. Dickey. SAS System for Forecasting Time Series. SAS Institute Inc. Cary NC. 1986.

[26] Walter Willinger, Vern Paxson, and Murad Taqqu, Self-similarity and Heavy Tails: Structural Modeling of Network Traffic, Adler, R., Feldman, R., and Taqqu, M.S., (editors), In A Practical Guide to Heavy Tails: Statistical Techniques and Applications, Birkhauser, 1998.

[27] Cox D., Long-Range Dependence: a Review. H. A. David and H. T. David (eds.), In Statistics: An Appraisal, Iowa State Statistical 
Library, The Iowa State University Press, 1984, pp.55-74.

[28] Duffy D.E., McIntosh A.A., Rosenstein M., Willinger, W., Statistical analysis of CCSN/SS7 traffic data from working CCS subnetworks, IEEE JSAC, Vol. 12, No. 3, April 1994, pp. 544551.

[29] Ashok Erramilli, Onuttom Narayan, Walter Willinger, Experimental queueing analysis with long-range dependent packet traffic, IEEE/ACM Transactions on Networking, Vol. 4, No. 2, April 1996, pp. 209 - 223.

[30] V. Bolotin, J. Coombs-Reyes, D. Heyman, Y. Levy and D. Liu, IP Traffic Characterization for Planning and Control, Teletraffic Sci. and Eng., vol. 3a, Proc. 16th Int'l. Teletraffic Congress, P. Key and D. Smith, Eds., June 1999, pp. 425-436.

[31] http://www-dirt.cs.unc.edu/unc02_ts/, visited on January 2005.

[32] Fei Xue and Lj. Trajkovic, Performance analysis of a waveletbased Hurst parameter estimator for self-similar traffic, Proc. SPECTS '2000, Vancouver, BC, Canada, July 2000, pp. 294-298.

[33] UNC Network Data Analysis Study Group, University of North Carolina, http://www-dirt.cs.unc.edu/net_lrd/, visited on December 2002.

[34] Stilian Stoev, Murad Taqqu, Cheolwoo Park and J.S. Marron, Strengths and Limitations of the Wavelet Spectrum Method in the Analysis of Internet Traffic, SAMSI, Technical Report \#2004-8, March 26, 2004.

[35] N. Cackov, Wavelet-based estimation of long-range dependence in video and network traffic traces, MSc thesis, School of Engineering Science, Simon Fraser University, 2005.

[36] Clegg Richard G., A practical guide to measuring the Hurst parameter, International Journal of Simulation: Systems, Science \& Technology, 7(2), 2006, pp. 3-14.

[37] Sonechkin D. M., Astafyeva N. M., Datsenko N. M., Ivachtchenko N. N. and Jakubiak, B., Multiscale Oscillations of the Global Climate System as Revealed by Wavelet Transform of Observational Data Time Series, Theoretical and Applied Climatology, Volume 64, Issue 1/2, 1999, pp. 131-142.

[38] M.S. Taqqu, V. Teverovsky, and W. Willinger, Estimators for long-range dependence: an empirical study, Fractals, 3, 1995, pp. 785-798.

[39] Trang D. D., SÁNDOR M., On the Effects of Non-Stationarity in Long-Range Dependence Tests, Periodica Pol. Elec. Eng., Vol. 43, No. 4, 1999, pp. 227-250.

[40] Marwan Krunz: On the Limitations of the Variance-time Test for Inference of Long-range Dependence, INFOCOM 2001, pp.12541260

[41] Thomas Karagiannis, Michalis Faloutsos and Rudolf Riedi, LongRange Dependence: Now you see it, now you don't! Global Internet Symposium (in IEEE GLOBECOM), Taipei, Taiwan, November 17-21, 2002

[42] S. Molnár, A. Vidács, On Modeling and Shaping Self-Similar ATM Traffic, 15th International Teletraffic Congress, Washington, DC, USA, June 23-27, 1997.

[43] Karim M. Rezaul and Grout, V., Exploring the Reliability and Robustness of HEAF(2) for Quantifying the Intensity of LongRange Dependent Network Traffic, International Journal of Computer Science and Network Security, Vol. 7, No. 2, February 2007, pp221-229.

[44] Karim M. Rezaul, Algirdas Pakštas, Robert Gilchrist, Thomas M. Chen, HEAF: A Novel Estimator for Long-Range Dependent Self-similar Network Traffic, Y. Koucheryavy, J. Harju, and V.B. Iversen (Eds.): Next Generation Teletraffic and Wired/Wireless Advanced Networking (NEW2AN), May 29 - June 2, 2006, LNCS 4003, pp. $34-45$.

[45] Karim M. Rezaul , Algirdas Pakštas, Robert Gilchrist, Investigation of the Properties of the HEAF Estimator Using Simulation Experiments and MPEG-encoded Video Traces, 10th IEEE International Conference on Intelligent Engineering Systems (INES 2006), London, UK, June 26-28, 2006, pp. 276-281.

[46] V. Paxson and S. Floyd., Wide Area Traffic: The Failure of Poisson Modeling, IEEE/ACM Transactions on Networking, June
1995, pp.236-244.

[47] Mark E. Crovella and Lester Lipsky, Long-Lasting Transient Conditions in Simulations with Heavy-Tailed Workloads, In Proceedings of the 1997 Winter Simulation Conference, 1997. pp.1005-1012.

[48] Mark E. Crovella and Azer Bestavros, Explaining World Wide Web Traffic Self-Similarity, October 12, 1995, Boston University, Technical Report TR-95-015.

[49] Karim M. Rezaul. \& Grout, V. A Comparison of Methods for Estimating the Tail Index of Heavy-tailed Internet Traffic, Proceedings of the second International Joint e-Conference on Computer, Information, and Systems Sciences, and Engineering (CISSE 2006), December 4 - 14, 2006

[50] Karim M. Rezaul and Algirdas Pakštas, Web Traffic Analysis Based on EDF Statistics, 7th Annual PostGraduate Symposium on the Convergence of Telecommunications, Networking and Broadcasting (PGnet 2006), Liverpool, UK, June 26-27, 2006, pp. 340-345.

[51] Karim M. Rezaul and Grout, V. CoLoRaDe: A Novel Algorithm for Controlling Long-Range Dependent Network Traffic, Proceedings of the Sixth International Conference on Networking (ICN 2007), Martinique, French Caribbean, 22-28 April 2007.

[52] Amit Aggarwal, Stefan Savage and Thomas Anderson, Understanding the Performance of TCP Pacing. Proc. of the IEEE INFOCOM 2000 Conference on Computer Communications, March 2000, pp. 1157 - 1165.

[53] K. Park, G. Kim, and M. Crovella, On the effect of self-similarity on network performance, In Proceedings of the SPIE International Conference on Performance and Control of Network System, November 1997, pp 296-310.

[54] Karim M. Rezaul and Grout, V. BPTraSha: A Novel Algorithm for Shaping Bursty Nature of Internet Traffic, Proceedings of the 3rd IARIA/IEEE Advanced International Conference on Telecommunications (AICT 2007), May 13-19 2007, Mauritius.

[55] R. Riedi, M. S. Crouse, V. Ribero, and R. G. Baraniuk, A Multifractal Wavelet Model with Application to Network Traffic, IEEE Trans. Info. Theory, Special Issue on Multiscale signal analysis and its application, Vol. 45, April 1999, pp992-1018.

[56] Jens Feder, Fractals, Plenum NY and London, 1988.

[57] G. E. P. Box and G. M. Jenkins, Time Series Analysis: Forecasting and Control, 2nd ed. San Francisco, CA: Holden Day, 1976.

[58] C. W. J. Granger and R. Joyeux, An introduction to long-memory time series models and fractional differencing, Journal of Time Series Analysis, vol. 1, pp. 15-29, 1980.

[59] J. R. M. Hosking, Fractional differencing, Biometrika, vol. 68, pp. 156-176. 1981

[60] D. Veitch, P. Flandrin, P. Abry, R. Riedi, R. Baraniuk. The Multiscale Nature of Network Traffic: Discovery, Analysis, and Modelling, IEEE Signal Processing Magazine, pp 28-46, Vol. 19, No. 3, May, 2002.

[61] Gao J. and Rubin I., Multiplicative Multifractal Modeling of Longrange dependent Network Traffic, International Journal of Communication Systems, 2001, Vol. 14, pp 783-801.

[62] Steven Bradley Lowen and Malvin Carl Teich, Fractal-Based Point Processes, Wiley, 2005

[63] Parulekar M., Makowski M. A., M|G|Infinity Input Processes: A Versatile Class of Models for Network Traffic, In Proc. IEEE INFOCOM, Kobe, Japan, pp419-426, April 1997.

[64] M. Krunz and A. M. Makowski. Modeling Video Traffic Using M/G/Infinity Input Processes: A Compromise between Markovian and LRD Models. In IEEE Journal on Selected Areas in Communications, 16(5): 733-748, June 1998.

[65] Marwan Krunz, Armand M. Makowski, A Source Model for VBR Video Traffic Based On M/G/infinity Input Processes. INFOCOM 1998, pp1441-1448.

[66] D. R. Cox and V. Isham, Point Processes, Chapman and Hall, New York, 1980. 